\title{
Turismo, viajes y lazos sociales: el caso de los turistas VFA en la ciudad de Buenos Aires
}

\author{
Bárbara Catalano* \\ Instituto de Investigaciones Gino German (Argentina)
}

\begin{abstract}
Resumen: El presente trabajo aborda una modalidad turística asociada a los viajes cuyos principales motivos son visitar amigos y/o familiares (VFA). La hipótesis radica en que el tipo de turismo vincular es una modalidad turística integradora, en términos de interacción social y sociabilidad en las estadías de los turistas en la ciudad de Buenos Aires. En primer lugar se exponen los lineamientos teóricos en los que se basa la conceptualización del turismo VFA. Luego, se busca describir y caracterizar esta modalidad turística en los últimos años, analizar la relación con los lazos sociales, los movimientos migratorios, la forma de desenvolverse y las prácticas sociales en el destino, para finalmente esbozar reflexiones en torno a la integración en términos de interacción social que presenta este tipo de turismo. La metodología es principalmente cualitativa basada en entrevistas semiestructuradas a turistas y actores clave, complementada con estadísticas para contextualizar el fenómeno.
\end{abstract}

Palavras Clave: Turismo; Viajes; Visitas; Vínculos; Movilidades; Amigos; Familiares.

Tourism, travel and social ties: the case of VFR tourist at Buenos Aires city.

Abstract: This paper addresses the sector of VFR tourism: visiting friends and relatives (VFR). The hypothesis is that tourism based on social ties facilitates integration in terms of social interaction and social identification in the destination, in this case the city of Buenos Aires. The concept itself of VFR tourism is dealt with together with a description and attempted characterisation of the exact movement of this type of tourism over the last few years as a result of migratory movements with emphasis on social ties and practices. The emphasis is on the degree of social integration and identification occurring in this type of tourism. The methodology used is qualitative, based on semi-structured interviews of key agents and is complemented with statistics designed to give fuller access to the comprehension of the context.

Keywords: Tourism; Travel; Visiting; Ties; Mobility; Friends; Relatives.

\section{Introducción}

Desde hace ya varias décadas el turismo presenta transformaciones desde la oferta, la demanda y también desde los enfoques teóricos para conceptualizarlo. Los avances técnicos, vinculados al transporte, a la tecnología y a las comunicaciones han acelerado esas transformaciones y actualmente estamos ante una vasta gama de destinos y modalidades turísticas. Tal es así que este trabajo se enfoca en una de las tipologías turísticas que es altamente practicada pero que escapa a la atención del sector privado y público. Su protagonista es el turista que visita a un familiar o amigo y en la literatura anglosajona se denomina turismo VFR o mercado VFR, por sus siglas en inglés visiting friends and relatives; y que en este trabajo llamaremos turismo cuyo motivo principal es la visita a un familiar o amigo, VFA.

A pesar de que los estudios sobre turismo y viajes se han intensificado y mejorado notablemente en los últimos años (Falero \& Campodonico, 2014), los turistas VFA, no aparecen con frecuencia en la literatura, más bien se los omite o se los pasa por alto. En líneas generales existe una preferencia por el tipo de turista consumista, estereotipado, predecible y ritualizado (MacCannell, 2003; Margulis, 2006; Watson \& Kopachevsky, 1994), que presenta regularidades a través de las cuales es posible generar

Instituto de Investigaciones Gino German (Argentina); E-mail: catalano81@gmail.com; https://orcid.org/0000-0002-7565-8451 
pautas de conductas que remitan a una teoría del turista. Sin embargo, el turista VFA representa la segunda modalidad de importancia en términos de participación del turismo receptivo, a nivel nacional e internacional, incluso es una de las formas más antiguas de viajar, y en los últimos años manifestó un incremento sostenido en términos de volumen de llegadas (Backer \& King, 2015).

Es por eso que en este trabajo se abordará este tipo de turismo asociado a los vínculos, partiendo del supuesto de que el turismo VFA constituye una modalidad turística integradora, en términos de interacción social y sociabilidad en las estadías de los turistas en el destino: ciudad de Buenos Aires, sobre todo por su relación con los vínculos y los lazos sociales. Para ello el objetivo principal versa en describir y caracterizar este tipo de modalidad turística en los últimos años, analizar la relación con los lazos sociales, los movimientos migratorios, la forma de desenvolverse y las prácticas sociales en el destino, para finalmente esbozar reflexiones en torno a la integración en términos de interacción social.

Esta modalidad está caracterizada porque el principal motivo del turista no reside necesariamente en visitar un lugar distinto, vacacionar o entretenimiento, sino que prima una motivación social y vincular. Desde una mirada sociológica, se concibe al turismo como un tipo de movilidad temporal, en el que las movilidades presentan un rol destacado, que dan sentido y construyen lugares (Cresswell, 2008; Sheller \& Urry, 2004). Según los autores, el turismo está asociado a los recuerdos, los cuerpos, las emociones, los entornos que reflejan distintas combinaciones entre movilidad, inmovilidad, trabajo, ocio, utopías e imaginarios. Se abordará al turismo desde un enfoque social donde las movilidades se sitúan en el centro de las estructuras sociales y éstas son concebidas como un entramado complejo de significaciones (Catalano, 2019). A su vez, se mostrará la relación particular entre el VFA, los flujos migratorios y la heterogeneidad de las movilidades actuales en la modernidad. En esa línea, los aspectos de conexión entre el turismo VFA y las migraciones (Backer \& King, 2015; Otero, 2009; Williams \& Hall, 2000) son visibles desde diversas escalas, a saber desde la global, en relación a las orientaciones y magnitudes de estos flujos, y también a partir de las motivaciones, interacciones y prácticas de los turistas con la población residente.

A modo de ordenamiento del presente trabajo, se introduce una reseña metodológica para poder situar en tiempo, espacio y alcance el objeto de estudio. Luego, se exponen los lineamientos teóricos que permiten comprender la conceptualización de la modalidad FVA desde el enfoque de la sociología del turismo. Posteriormente, se ahonda en una caracterización sobre la representatividad de esta modalidad en la Argentina, teniendo en cuenta su importancia en términos de participación en relación al total del turismo receptivo internacional. Se esbozan líneas de conexión entre el turismo VFA y las migraciones, ya que como se observa en la revisión de la literatura previa, cobra particular protagonismo el nexo o familiar al que vienen a visitar los turistas. Se definen los indicadores a través de los cuales se dimensiona la integración social de los turistas VFA, interacciones y socialización. Luego, ya en base al análisis de los datos recolectados se efectúa una caracterización del VFA y se analizan los niveles de interacción y sociabilización de estos turistas con la comunidad local. Finalmente, se esbozan reflexiones sobre la importancia de este tipo de turismo, que suele ser invisibilizado y no priorizado por la industria turística, pero que constituye un fenómeno de importancia en un contexto de movilidades cada vez más frecuentes y de aceleración y diversificación de los viajes.

\section{Aspectos metodológicos}

Cabe destacar que este trabajo se realizó en el marco de una investigación mayor, que desembocó en una tesis doctoral y que implicó la realización de un trabajo de campo y recolección de datos a través de entrevistas semi-estructuradas. El total de entrevistas fue de 52, de los cuales 24 se realizaron a informantes clave y 28 a turistas ${ }^{1}$, entre noviembre de 2015 y diciembre de $2016^{2}$. Para la muestra se seleccionan los turistas regionales no residentes cuyas nacionalidades de origen son Brasil, Uruguay, Paraguay y Chile.

Se optó por priorizar el método cualitativo con el fin de comprender el análisis de las estructuras, los procesos sociales y las relaciones involucradas en la implementación de las acciones y la visión de los actores diferenciados (De Souza Minayo, 2009, p. 295). A menudo resultan insuficientes las fuentes para explicar la significación de los viajes, como parte esencial de la vida social y como fenómeno inherente a la comprensión de la sociedad moderna. Como consecuencia, se implementan nuevas formas de pensar y analizar los viajes y las interacciones que se dan en estos, principalmente a través de metodologías plurales y heterogéneas (Merriman, 2014, p. 168). Para el caso del turismo, como una forma de movilidad, se necesita de la comprensión del fenómeno como movimiento humano dentro de un espectro mucho 
más amplio de las movilidades sociales y físicas. Aquí se sitúan las movilidades en el centro de las estructuras sociales, se estudia a los turistas que se movilizan, interactúan y actúan en los espacios públicos; concibiéndolos como sujetos sociales que reflejan significados, experiencias, ritmos y prácticas (Cresswell, 2008). En cuanto al análisis de datos, se consideran las relaciones y experiencias turísticas, no a modo de formulaciones vacías que silencian a los propios protagonistas, sino reconociendo el conjunto de representaciones, significaciones y sentidos que generan los sujetos como parte de un conjunto social (Achilli, 2014). Además, se utiliza el microanálisis incluyendo la codificación axial y abierta que permiten hacer uso de las múltiples técnicas analíticas, como también examinar e interpretar datos de manera cuidadosa y minuciosa (Strauss \& Corbin, 2002). Finalmente, se evalúan las variaciones, impulsando el proceso inductivo y se efectúan comparaciones entre los distintos relatos de los entrevistados arribando a conclusiones sobre lo que producen las prácticas de estos sujetos en el destino y en las relaciones sociales.

\section{Conceptualizando el turismo que visita a un familiar y/o amigo (VFA)}

Para definir al turismo vincular es necesario primero ahondar sobre el turismo como un tipo de movilidad y sobre los vínculos en general. En los últimos años las movilidades se han intensificado en volumen y en diversidad, generando transformaciones en las instituciones, y en los regímenes que las conducen y organizan (Larsen et al., 2006). El turismo, como un fenómeno social es interpretado como un intercambio de sociedades (Lanfant, 1980). Se alteran los patrones y se adquieren nuevos significados de los viajes, del turismo y de las migraciones; las familias se reconfiguran y la vida social se transforma a partir de los lazos emergentes producto de las nuevas movilidades. La proximidad, la conectividad y la temporalidad de las familias presentan giros que requieren un repensar las relaciones entre los viajes en la modernidad (Glick Schiller \& Salazar, 2013; Hannam et al., 2006; White \& White, 2007).

El turismo vincular hace alusión a las circunstancias que impulsan el desplazamiento de un sujeto hacia un territorio distinto al de su lugar de origen cuyos motivos principales radican en la visita a un familiar y/o amigo. En este sentido, cabe realizar la distinción entre los lazos familiares, de amistades y/o cualquier otro tipo de afinidad que implique un vínculo con otra persona en el destino.

Conceptualizaciones significativas desde las ciencias sociales, sobre este tipo de turismo son realizadas por Hall, C. M., \& Williams (2001) cuya obra aborda la intrínseca relación e interdependencia entre turismo y migraciones. Los autores plantean que definir al turismo y a las migraciones implica una compleja y hasta tramposa tarea; sin embargo, se precisa de aproximaciones conceptuales iniciales, para luego ir mejorando y precisándolas.

En esa línea se define al turismo VFR, por sus siglas en inglés visiting friends and relatives, como una modalidad turística para la cual la cual la migración es una condición previa, que puede ser directa o indirecta, es decir, de generaciones anteriores. A pesar de ello se aclara que esta relación no es excluyente, incluso admiten que puede llegar a interpretarse como una simplificación excesiva, ya que pueden intervenir otras razones a la hora de decidir el desplazamiento hacia un lugar de destino ${ }^{3}$, tales como las atracciones con las que cuenta el lugar donde reside el familiar y / o amigo, o el ahorro de dinero en el costo del alojamiento.

En esa línea, es clave destacar que este tipo de turismo suele estar subestimado y conlleva un área de vacancia para la academia, además que representa un segmento poco rentable para el sector privado (Backer \& King, 2015). A pesar de ello, detrás de esta modalidad se enlazan numerosos procesos sociales pasibles de ser estudiados, sobre todo, a partir de casos de estudio, como puede observarse en el turismo de diáspora o en la transformación que sufren las sociedades anfitrionas a partir de la visita de sus allegados (Coles et al., 2006; De Santana Pinho, 2018; Shani \& Uriely, 2012).

En ese sentido, si bien en este trabajo abordaremos la generalidad del turismo (VFA), es posible desmembrar esta categoría en distintas sub-modalidades asociadas a tipos de vínculos que difieren entre sí, tales como familiar que migró para mejorar la calidad de vida, familiar que migró para realizar sus estudios en el exterior, un amigo que ha conocido en un viaje, un amigo que decidió radicarse en el destino por otro motivo, solo para mencionar algunos ejemplos. Williams and Hall (2001) describen algunas de las sub-modalidades asociados a motivos tales como: visitar a un familiar, conocer a un nuevo integrante de la familia que acaba de nacer, compartir ciertas celebraciones con allegados que residen en otro lugar distinto al del visitante. Asistir a cumpleaños, casamientos y funerales de familiares y o amigos. Es así como se intenta, por un lado definir esta modalidad de turismo, sin perder de vista ciertas características particulares que pueden observarse en las diversas prácticas de movilidad se observan en la realidad. 


\section{La modalidad VFA y su representatividad}

A partir de la conceptualización previa es factible dimensionar el volumen que representa este turismo en un destino determinado. En el caso de Argentina ello se deduce de la ETI ${ }^{4}$, que provee información que propicia un acercamiento hacia este tipo de turismo, en particular en la pregunta por motivación ${ }^{5}$, dado que una de las opciones disponibles para seleccionar es: visitar amigos y/o familiares. Para el caso de Argentina, por ejemplo, según la metodología implementada por el MINTUR e INDEC a través de la encuesta ETI se definen los siguientes conceptos sobre los cuales se indaga a los turistas internacionales al momento de ingresar y egresar al país. Así, la tipología por $\mathrm{VFA}^{6}$ está dentro del segundo motivo luego de "ocio, vacaciones y recreación”. Como se observa a continuación, los viajes por esta motivación se han incrementado en los últimos años, no solamente en términos absolutos, sino también aumenta la proporción de visitantes que vienen por este motivo en detrimento de los otros. Si caracterizamos al turismo de ocio y vacaciones como una modalidad tradicional y estandarizada, la predominancia de otro motivo podría traducirse en la fragmentación y diversificación de la oferta y demanda turística, lo que en ocasiones se define como pos-turismo, actividad propia del capitalismo desorganizado, y que se ve reflejado en los cambios de las pautas de movilidad, observadas en formas de viajar más diferenciadas y fragmentadas (Lash \& Urry, 1998).

Esta modalidad VFA ha ido aumentando en el total receptivo internacional a lo largo de los últimos años, como se observa en los datos provenientes de la ETI en la que se muestran las llegadas de turistas a la Argentina según motivación. A partir del cuadro siguiente es factible inferir que existe una mayor diversificación de los motivos de viaje, lo que se corresponde con los argumentos previos en torno a la pluralidad de las formas de movilidad y a un desinterés cada vez mayor por el turismo estandarizado.

\section{Cuadro 1: Llegadas de turistas internacionales a aeropuerto internacional de Ezeiza y Aeroparque ${ }^{7}$ según motivo de viaje entre 2006-2015}

\begin{tabular}{|c|c|c|c|c|c|c|c|c|c|c|c|}
\hline & & 2006 & 2007 & 2008 & 2009 & 2010 & 2011 & 2012 & 2013 & 2014 & 2015 \\
\hline \multirow{5}{*}{ 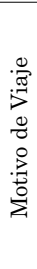 } & $\begin{array}{l}\text { Vacaciones Ocio } \\
\text { Recreación }\end{array}$ & 1.339 .590 & 1.401 .224 & 1.460 .872 & 1.180 .259 & 1.616 .549 & 1.601 .453 & 1.627 .607 & 1.443 .747 & 1.372 .886 & 1.232 .598 \\
\hline & VFA & 345.185 & 413.465 & 408.668 & 414.142 & 419.489 & 457.653 & 625.197 & 623.084 & 755.025 & 711.414 \\
\hline & $\begin{array}{l}\text { Negocios (conferencias/ } \\
\text { Congresos/ } \\
\text { exposiciones/otros) }\end{array}$ & 361.881 & 467.862 & 435.067 & 388.276 & 486.866 & 500.562 & 483.640 & 511.495 & 540.692 & 560.456 \\
\hline & Otros & 100.030 & 104.636 & 100.364 & 99.922 & 125.056 & 132.464 & 82.824 & 97.520 & 82.538 & 66.951 \\
\hline & Estudio & & & & & & & 85.184 & 85.717 & 77.630 & 68.975 \\
\hline
\end{tabular}

Fuente: Elaboración propia en base a datos provistos por MINTUR.

\section{Gráfico 1: Llegadas de turistas internacionales a aeropuerto internacional} de Ezeiza y Aeroparque según motivo de viaje entre 2006-2015

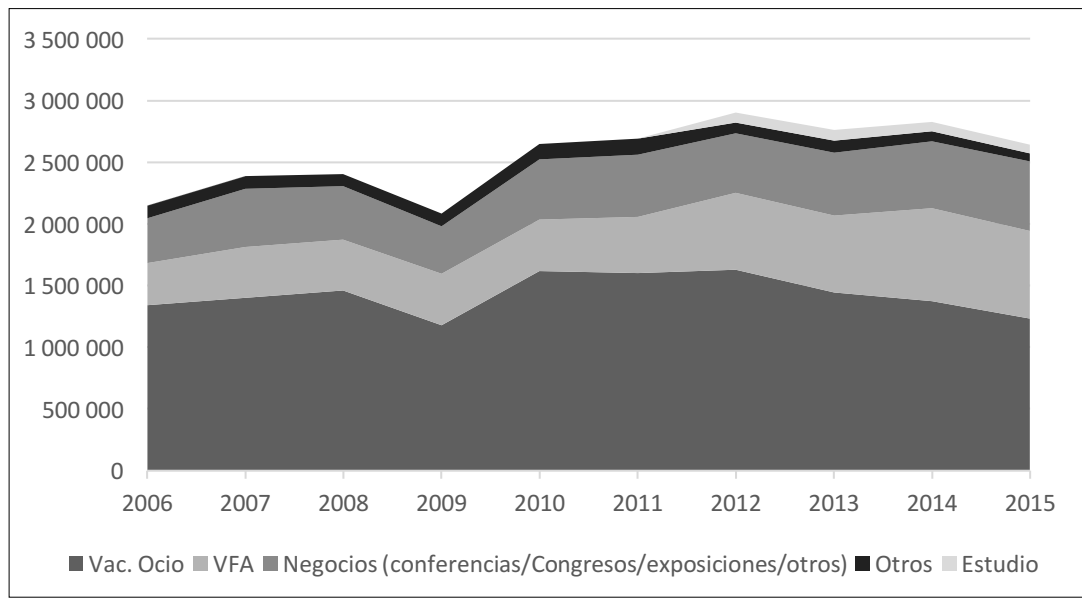

Fuente: Elaboración propia en base a datos provistos por MINTUR. 
Más allá de las consideraciones sobre la representatividad de esta modalidad y de las distinciones entre nacionalidades de origen del turismo vincular, se precisa de abordar una dimensión que emerge como determinante en el desenvolvimiento de este turista y que se corresponde también con las lógicas de las movilidades desde una escala global. Por esa razón es que se analizará a continuación algunas líneas de conexión entre el turismo y los flujos migratorios.

\section{Flujos, movilidades y turismo}

Tal como se mencionó al inicio, varios autores destacan la conexión entre las migraciones y el turismo, sobre todo en lo que respecta al turismo VFA (Backer \& King, 2015; Hall \& Williams, 2001). Es posible afirmar que las direcciones de los flujos de turistas y migrantes suelen ir en contramano (Barretto, 2009), es decir si se observara a grandes rasgos las direcciones de estos movimientos, los primeros se dirigen desde países más desarrollados hacia menos desarrollados, mientras que los segundos se orientan principalmente hacia países más desarrollados de los de su residencia original (Vellas, 2004). En ese mismo sentido, Cordero Ulate (2006) sostiene que el turismo es una actividad dirigida hacia los países pobres, como es el caso de los países del Caribe, aunque ello fue cambiando en los últimos años, sobre todo por la democratización del bienestar que afectó de manera particular a los derechos de los trabajadores y al acceso al ocio.

Es necesario realizar diferenciaciones asiduas entre turismo, residencia y migraciones, teniendo en cuenta las condiciones que aplicarían a cada fenómeno, sobre todo en relación al tiempo de permanencia en un sitio determinado que no es el del origen habitual y el móvil. Por ello se precisa distinguir entre las motivaciones que impulsan la movilidad hacia un destino, el momento de vida de los sujetos que se desplazan, la perspectiva biográfica y las relaciones sociales que surgen a raíz de estos intercambios $\left(\right.$ Huete, 2009) ${ }^{9}$. La autora efectúa un acercamiento conceptual hacia la relación compleja entre migración y turismo sobre todo desde las implicancias legales y burocráticas de los individuos identificados como "turistas" o "inmigrantes".

De este modo existen varias relaciones entre el turista que viene a visitar a algún amigo o familiar y otro tipo de movilidades como son las migraciones (Hall \& Williams, 2001), dado que los migrantes que se constituyen en un determinado destino son elementos que propician la movilidad de estos turistas hacia visitar el destino ${ }^{10}$. De todas formas, esta relación no es excluyente dado que en ocasiones las personas que vienen a visitar estos turistas no necesariamente son de la misma procedencia de ellos ni familiares. Es posible que ellos vengan a visitar a algún amigo que hayan conocido en algún otro viaje o bien a algún familiar político. A su vez, el tipo de turismo VFA está conectado con el tipo de turismo de estudio y la movilidad académica (Curcio \& Luna, 2019; Mayer \& Catalano, 2018) ya que suelen permanecer estadías extensas, lo que provoca que sus familiares los vengan a visitar.

\subsection{Población extranjera en Argentina}

$\mathrm{Al}$ observar las poblaciones de migrantes en Argentina y en particular en Buenos Aires se encuentran algunas particularidades que contribuyen al entendimiento de la composición poblacional en la que se encuentran los nacidos en Argentina y en el exterior. Los flujos migratorios se insertan en los diversos procesos de integración regional como dinámicas que articulan la integración productiva y social. El Mercosur, bloque de integración en el que Argentina se encuentra inserta, no está exento y los movimientos migratorios emergen como un aspecto sociocultural que profundiza la unión, teniendo en cuenta además la histórica cultura compartida de sus pueblos (Modolo, 2012; Novick, 2010).

Según los censos poblacionales de los años 2001 y 2010 se observa que la población migrante de Argentina no solamente creció en términos absolutos sino también en proporciones dado que para el 2001 los habitantes migrantes representan un 11\% mientras que para el año 2010, un 13\%. La población de Buenos Aires muestra un aspecto cada vez más plurinacional a raíz de un mayor número de extranjeros que viven en la ciudad, aspecto que guarda cierta correspondencia con los movimientos poblacionales de aquellas personas que vienen a visitar a sus familiares y más aun considerando la apertura y cosmopolitismo que caracteriza a la ciudad, como también a sus características de ciudad global (Bertoncello \& Troncoso, 2014, p. 10; Catalano, 2018). 
Cuadro 2: Población ciudad de Buenos Aires censos 2001 y 2010 según lugar de nacimiento

\begin{tabular}{|l|c|c|c|}
\cline { 2 - 3 } \multicolumn{1}{c|}{} & \multicolumn{2}{c|}{ Lugar de nacimiento } & \multirow{2}{*}{ Total } \\
\cline { 2 - 3 } \multicolumn{1}{c|}{} & En el país & En el extranjero & \\
\hline Año 2001 & 2.459 .399 & 316.739 & 2.776 .138 \\
\hline Porcentaje & $89 \%$ & $11 \%$ & $100 \%$ \\
\hline Año 2010 & 2.508 .373 & 381.778 & 2.890 .151 \\
\hline Porcentaje & $87 \%$ & $13 \%$ & $100 \%$ \\
\hline
\end{tabular}

Fuente: elaboración propia en base a INDEC

Como se mencionó anteriormente dado que esta modalidad se asocia en gran medida a los flujos de migrantes a continuación se detallan las variaciones de poblaciones de migrantes de las nacionalidades de turistas regionales.

Para el caso de la población cuyo lugar de nacimiento es Brasil se observa un importante crecimiento entre los dos censos $78 \%$. A su vez, también se ha incrementado la representatividad de esta nacionalidad en el total de población de la CABA. Tal es así que para el año 2001 representaban un 0,2 \% mientras que para el 2010 un 0,4 \%. Esto se traduce en una mayor población de procedencia de Brasil. Sobre la población extranjera cuyo lugar de nacimiento es Chile no se han observado tantos cambios entre los dos censos dado que la variación porcentual interanual fue de un $2 \%$ en ascendente. Para el caso de Paraguay, al igual que con los brasileros que se asentaron en la ciudad se observa un significativo crecimiento de esta población que se traduce en un $71 \%$ de incremento y un aumento en la representatividad en el total de la población que va desde 1,7 en el 2001 a un 2,8 en el 2010. Finalmente el caso de Uruguay las cifras presentan otro tinte, dado que se observa una merma del $12 \%$ en los habitantes de esta nacionalidad lo que a su vez se refleja en una baja en la representatividad que va desde un 1,3\% en 2001 hasta un $1.1 \%$ para el año 2010. Cuadro 3: Población extranjera de países limítrofes en CABA, según origen,
variación intercensal y representatividad de la población total, 2001 y 2010

\begin{tabular}{|l|c|c|c|c|c|}
\cline { 2 - 6 } \multicolumn{1}{c|}{} & \multicolumn{3}{c|}{ Población migrante según lugar de origen } & $\begin{array}{c}\text { Representatividad del total de } \\
\text { la población de CABA }\end{array}$ \\
\hline & $\mathbf{2 0 0 1}$ & $\mathbf{2 0 1 0}$ & VAR \% & $\mathbf{2 0 0 1}$ & $\mathbf{2 0 1 0}$ \\
\hline Bolivia & 50.111 & 76.609 & 53 & 1,8 & 2,7 \\
\hline Brasil & 5.819 & 10.357 & 78 & 0,2 & 0,4 \\
\hline Chile & 9.648 & 9.857 & 2 & 0,3 & 0,3 \\
\hline Paraguay & 46.928 & 80.325 & 71 & 1,7 & 2,8 \\
\hline Uruguay & 34.750 & 30.741 & -12 & 1,3 & 1,1 \\
\hline
\end{tabular}

Fuente: elaboración propia en base a INDEC

Lo que se acaba de presentar tiene el fin de contribuir al conocimiento contextual en el que se desarrolla esta modalidad de turismo VFA y su vinculación particular con la población migrante registrada en los últimos censos. Es pertinente el abordaje meticuloso sobre esta modalidad dado que en ella intervienen muchas otras variables como ser las relaciones y vínculos a distancia y los lazos que se generan ya sea en instancias de presencia física como virtual.

\section{Repensando el turismo: lo social como móvil del viaje}

$\mathrm{Al}$ ahondar sobre el turismo VFA aparece la necesidad de repensar sus definiciones y conceptualizaciones que predominaron en los últimos años. Muchos de los estudios sobre el turismo conciben la práctica 
turística desde una perspectiva homogenea (Falero \& Campodonico, 2014; Fernández Miranda, 2011; Tribe, 2011) pasible de ser discutida, no solo desde las estadísticas sino también desde las múltiples modalidades turísticas que conviven en la realidad actual. Dadas las características cambiantes que afrontan los viajes en los últimos años deviene necesario repensar la conceptualización sobre el turismo, desde la práctica, la teoría y los modos de abordaje.

En ciertos contextos se observa la influencia del consumo en el turismo sobre todo en el re-direccionamiento de las prácticas turísticas hacia una cultura consumista, moldeada por la lógica del capitalismo y la mercantilización de la vida social moderna ${ }^{11}$ (Watson \& Kopachevsky, 1994). En ocasiones esa forma de turismo asociada el consumo también se ve reflejada en la estandarización y despersonalización de la actividad (Smith, 1989, p. 29), como también en una visión trivial del turismo a través de la cual se evita problematizarlo.

En otro orden, la modalidad VFA implica hacer foco en el turista asociado a los vínculos, a los compromisos familiares y al afecto. En la motivación del turista prima una motivación social y vincular. Ahora bien, por motivo de viaje existen varias acepciones, por un lado se encuentra la determinación metodológica de los organismos gubernamentales, tales como OMT ${ }^{12}$ o MINTUR ${ }^{13}$, que está basada en una la encuesta de turismo internacional que se realiza en los aeropuertos y otros puntos de ingresos al país y donde se realiza la siguiente pregunta a los turistas "¿cuál es el motivo principal de su viaje?" ${ }^{4}$. Pero, por otro lado, también radica en real sentido de los actos de las personas dado que el turismo es un fenómeno social y como tal es comprensible si puede reducirse a acciones humanas y a estas se las hace comprensible ahondando en sus motivos conducentes (Schutz, 2003, p. 25). Las prácticas suelen repetirse en usos y costumbres, reflejando motivaciones intrínsecas a esas acciones. Se añade a ello la concepción de los motivos conducentes que delimitan los cursos de acción, que dan sentido a la acción (Schutz, 2008, p. 89) y que implican genuinas relaciones de causalidad.

De este modo, la sociabilización en el turismo, basada en situaciones de interacción (Giddens, 1995) conducen hacia la integración social, construyendo lazos o siendo puente para profundizar el conocimiento sobre la cultura y sociedad que se está visitando. Los lazos sociales permiten comprender los entramados societarios e intersubjetivos de los individuos que comparten ciertos ámbitos diversos como puede ser el laboral (Murmis \& Feldman, 2002) o los ámbitos de socialización propios de las experiencias turísticas, dado que son entornos en los que pueden propiciarse la construcción de vínculos con el otro. Por ello es que se utilizan estas dimensiones de análisis como ejes estructurales del trabajo de campo donde se indaga sobre la socialización en los viajes turísticos.

\section{Los turistas VFA en la ciudad de Buenos Aires}

Para elucidar si este tipo de turismo facilita las situaciones de integración se analiza su desenvolvimiento en el destino turístico, en este caso, la ciudad de Buenos Aires. Uno de los elementos esenciales de cualquier práctica turística, es el recorrer y visitar en el destino visitado, por eso es que aquí se interpela a los sujetos con el fin de elucidar las características que entrañan las diversas prácticas del turismo VFA.

La forma de conectarse con el destino y de ahondar en el conocimiento sobre la cultura local por parte de los turistas VFA es distinta a la de las otras modalidades de turismo ${ }^{15}$. En estos casos los turistas son orientados por una persona conocida, quien los está alojando y en quien confían. Los anfitriones, diagraman los paseos, invitan a los visitantes a eventos sociales propios de la vida cotidiana del residente y de este modo se propician mayores situaciones de encuentros y sociabilización entre turistas y residentes. Esta es una forma de pasear que alejada de los circuitos comerciales atravesados por la intermediación turística, lo que decanta en tipos de paseos impredecibles y heterogéneos.

Sin embargo, es posible dilucidar algunas propiedades de esta categoría considerando las entrevistas a informantes nexos que fueron anfitriones de estos turistas. El elemento territorial indispensable en los estudios turísticos no escapa a este abordaje, por lo que el espacio por donde se desplazan y circulan los turistas se diferencia del turista tradicional. Además los recorridos implican una extensión de territorio mucho mayor, dado que los anfitriones llevan a los turistas amigos hacia lugares que no son turísticos y a barrios que exceden el tránsito típico turístico, como se observa en el fragmento siguiente: 
- ¿Ellos te preguntan a dónde ir? (refiriéndose a los turistas que vienen a visitar al anfitrión)

- La familia no, lo dejan en mis manos. Yo les organizo el viaje. En el primer viaje los llevo a visitar los lugares típicos, Recoleta, San Telmo, La Boca. Las veces siguientes los llevo a lugares no tan turísticos y que me gusta a mí... Por ejemplo Sarquis o una parrilla de barrio. Cosa que el turista no hace.

- Con mi familia ahora cuando viene hacemos mucha cosa de conocer barrios, pasear, etc. Caminar por Almagro $^{16}$ durante la tarde...Ir a algún barcito más alternativo de ahí... salir un poco del circuito turístico, Palermo, Recoleta. [...] Los que ya vinieron varias veces, les gusta conocer, cosas que salen de lo turístico. Ir a comer a un barrio, Villa Devoto, por ejemplo. Como para conocer una parte diferente de la ciudad. [...] Lo que me cambio la vida con las visitas es la bicicleta, me encanta la bicicleta. En los últimos años, con esa popularización de la bici, me ayudó mucho porque los agarro y vamos los llevo, todo el que viene está en el programa de bici. Y agarro la bicisenda y vamos conociendo la ciudad, por los distintos recovecos. (Nexo de turistas brasileros VFA, entrevista realizada el 9 de marzo de 2016)

En ese relato se observa cómo el conocer la cultura local es mucho más amplio y divergente que en las otras modalidades, justamente porque no es que no recorren lo típico, sino porque además de eso conocen otras zonas y otros barrios y sitios que son novedosos y atractivos para el público en general y para un turista en particular.

Otro de los elementos característicos de este tipo de turismo es el alojamiento, por lo general suelen alojarse en las casas o departamentos de la persona que vienen a visitar, lo que también acerca la relación con la vida cotidiana de la ciudad. Además los recorridos de los turistas no son fijos o estandarizados como podrían ser cuando siguen una guía turística o como cuando van al centro de información al turista y les recomiendan los lugares típicos. Por el contrario, en este caso los recorridos son flexibles y variados, se tiene en cuenta lo que desea el turista pero también una visión del anfitrión que se aleja a la lógica comercial del turismo y que facilita un conocimiento más genuino del lugar. Tal como se observa en el siguiente fragmento.

$-i$ Te vinieron a visitar?

- muchas veces, gente que conocí en viajes y familia.

- ¿los hospedabas?

- siempre en mi casa

- ¿vos los guiabas?

- más o menos hacía lo que hicieron conmigo al principio.

- ¿ellos ya tenían algo en mente o vos los llevabas directamente?

- sí, más o menos íbamos hablando, íbamos viendo. Pero siempre empezaba con lo clásico: Plaza de Mayo, Cafe Tortoni, algo en San Telmo, Algo en Recoleta, también algo cerca de casa, Homero Manzi, algún bodegón que me gusta. También los llevaba por lugares y barrios que no son muy conocidos. (Nexo de turistas ${ }^{17}$ brasileros VFA, entrevista realizada el 7 de julio de 2016)

En ese sentido se destaca la libertad y apertura que manifiesta este tipo de recorrer y que se distingue en gran medida del turismo tradicional. De hecho, en ocasiones estos turistas dicen que no son turistas, es decir, no se adjudican la característica de tal por realizar actividades fuera del común denominador, por movilizarse por lugares que no son los típicos turísticos y por tomarse colectivos o consumir productos tradicionales.

Tal es así que los turistas VFA participan de eventos sociales que no son propiamente turísticos sino son parte de la vida cotidiana de su nexo, tales como reuniones de amigos, cumpleaños, salidas con compañeros de trabajo o bien acompañarlos al supermercado o a la lavandería. El hecho de que los turistas tengan un vínculo previo en el lugar de destino amplía las posibilidades de generación de otros vínculos con personas con las que interactúe en un plano no turístico sino más bien relacionado a la cotidianeidad de los residentes.

Por el contrario, no pareciera constituir algo habitual la construcción de lazos entre el tipo de turismo más estructurado, y vacacional (Catalano, 2018), y en las ocasiones en que esto ocurre queda en la memoria de los turistas como un marcador sobre la visita, como aquello que resalta y que vale la pena mencionarlo como una anécdota del viaje. Ahora bien, los lazos sociales a veces son fortuitos, y a veces intercede un lazo previo o un ámbito de sociabilidad que los propicia.

Los lazos posteriores a las experiencias de los viajes también son inductores de los viajes, es decir el vínculo y la continuidad y enriquecimiento de este puede representar uno de los principales motivos que desencadena la acción del viaje a un determinado destino. El viaje permite la proximidad física 
y el ejercicio de prácticas comunicacionales y presenciales que no se dan de otra forma que no sea en situaciones cara a cara, y si bien la tecnología ha influido en las formas en las que estos lazos se desarrollan y perpetúan, existe una condición irremplazable del contacto real (Urry, 2002) ${ }^{18}$. De este modo la sociabilización, vínculos y lazos sociales son elementos causales y consecuentes de los viajes VFA y constituyen formas de relacionamientos entre partes.

\section{Integración en términos de interacción social}

La modalidad de turismo VFA presenta una íntima relación intermediada con un nexo, que suelen ser migrantes y la búsqueda de proximidad es el móvil del desplazamiento del turista hacia ese destino. A diferencia de los turistas tradicionales o los vacacionales ${ }^{19}$ Existe un vínculo cercano con un nexo que reside en el lugar de destino, quien no solamente facilita y provee de salidas y paseos más acordes a la cotidianeidad del lugar sino que también involucra a los turistas con personas que están por fuera del circuito turístico convencional y de la industria turística, generando un contacto más genuino con la cultura local y creando ámbitos de sociabilidad acordes a los mismos ciudadanos. De este modo, de los datos recopilados en el trabajo de campo se observa un alto nivel de interacción, que se debe a factores coyunturales como puede ser el entorno y ambiente en el que se desenvuelve el turista, pero también a las subjetividades correspondientes al sentido que para el turista implica el relacionamiento y el aspecto "social" en sus viajes.

Otro de los elementos que contribuyen a considerar este turismo como integrador es la estadía promedio de permanencia en el destino. Al analizar los datos estadísticos de la ETI se observa que esta modalidad presenta una cantidad de días en el destino mayor al resto de las modalidades. Exceptuando la modalidad por estudio, el motivo visita familiares y / o amigos es la segunda modalidad con la mayor cantidad de días de permanencia en el destino, casi 19 días frente a los casi 9 días que presenta la modalidad de vacaciones.

\section{Cuadro 4: Estadía promedio de los turistas no residentes por motivo de viaje. Año 2015}

\begin{tabular}{|l|c|}
\hline \multicolumn{1}{|c|}{ Motivo de viaje } & Estadía media en noches \\
\hline Vacaciones, ocio y recreación & 8,7 \\
\hline Visita a familiares y/o amigos & 18,6 \\
\hline Negocios (conferencias, congresos, ferias y exposiciones) & 5,8 \\
\hline Otros Negocios & 8,2 \\
\hline Estudio & 47,2 \\
\hline Otros & 14,1 \\
\hline
\end{tabular}

Fuente: Elaboración propia en base a AET 2015 - MINTUR

A medida que aumenta la cantidad de días de permanencia los visitantes alcanzan mayores grados de relacionamientos y lazos, por lo que esta modalidad de turismo aparece como la que propicia mayores niveles de integración en relación a las otras. Este aspecto también se ve observado en los relatos de las entrevistas, en los cuales los turistas esgrimen que debido al tiempo que permanecen en el destino pueden alcanzar otro tipo de conexión con la sociedad y el lugar. A ello se le añade que el turista VFA presenta una tasa de retorno mayor al tipo de turista convencional, por lo que se infiere un mayor relacionamiento con el lugar visitado a través del aprehendizaje acumulado en la sumatoria de las experiencias en las que el visitante tuvo contacto con lo local.

A su vez, según la mayoría de los relatos de los informantes clave, el turista VFA es "invisible", dado que escapa a los circuitos impuestos por la industria turística, y de hecho, no es de su agrado que lo denominen turista, por lo que intenta hacer todo lo posible para no hacer las típicas cosas que son habitualmente asociadas a las prácticas turísticas. Busca integrarse y la forma en la que lo alcanza es inmiscuirse entre la sociedad y quiebra la distinción que desde el análisis teórico se le adjudica a la sociedad receptora o comunidad local. 
El análisis de la integración en términos de interacción social al interior de esta modalidad conduce hacia una reflexión sobre el rol superlativo que presenta lo "social" en la totalidad de la experiencia turística. En esa línea, se observó que las relaciones que emergen de manera espontánea y natural en los viajes desencadenan una experiencia genuina de reciprocidad.

Además de la proximidad y el arraigo con los lazos sociales, los turistas también aprovechan para conocer el lugar y pasear. Hay una combinación entre el móvil social y el recreacional y se observa una intensidad significativa sobre las relaciones sociales de esta modalidad. Los turistas VFA presentan una forma de relacionarse con la sociedad receptora menos distante que otros tipos de turistas, dado que los nexos, que pueden ser familiares, amigos o bien conocidos, los insertan en ámbitos de socialización en los que ellos son parte, permitiendo de este modo una interiorización y conocimiento sobre diversos aspectos en los que hay una alimentación mutua de los acervos de conocimiento sobre la cultura del "otro".

Esta preeminencia del aspecto social en el turismo, ha sido denominada por ciertos autores como "des-exotización del viaje", haciendo alusión a que cada vez existen más motivos distintos al clásico: "búsqueda de lo exótico" (Larsen et al., 2007, p. 245), lo que revela un cambio de paradigma en las concepciones de los viajes. Este fenómeno puede explicarse también desde la ampliación de la ciudadanía global, el progreso en las comunicaciones y los medios de transporte, y sobre todo de la transformación del mundo en una comunidad interconectada en la cual cada miembro depende de otro (Urry, 2000) y en el que los turistas y viajeros están insertos en una constelación de flujos globales.

\section{Reflexiones finales}

A modo de reflexión final cabe destacar que este tipo de turismo refleja no solo una forma invisible de pasear, recorrer y viajar sino que también desentraña las complejas estructuras de la sociedad moderna, las escalas, los regímenes de movilidades y la importancia que tiene la proximidad y los lazos sociales en el turismo.

Así, sale a la luz la intensa correlación entre los flujos migratorios y los flujos turísticos en general y el tipo de turismo VFA en particular, sobre todo por los "nexos" que son los que motivan el desplazamiento de los turistas. En el caso de los turistas en la ciudad de Buenos Aires, aquellos denominados VFA o turistas vinculares, presentan situaciones y prácticas de interacción y sociabilidad significativas. Esos aspectos de vinculación social no se observan en otras modalidades turísticas, lo que conduce a afirmar la hipótesis inicial basada en que el turismo VFA es una modalidad turística integradora.

Es por ello que al analizar esta modalidad de viaje es que se reflexiona sobre la redefinición de turismo acorde a las vicisitudes del mundo contemporáneo, y a la diversificación del turismo, que se presenta en la actualidad, bajo formas cada vez menos estandarizadas. En el sistema capitalista actual y en las sociedades mercantilizadas, se puede pensar en un reflotar de los afectos y de los vínculos como móvil de un turismo desestructurado. En esa línea, nos replanteamos la configuración de los viajes actuales, mientras que emerge el interrogante: jestamos atravesando un escenario en el que hay menos turismo de masas y más viajes asociados a lo auténtico, a los sentimientos y a las emociones?

Esta es la forma des-exotizada del turismo y que en este trabajo se ha demostrado que contribuye a aunar las relaciones entre anfitrión e invitado o entre sociedad de origen y de destino, ya que se promueven situaciones de interacción e integración que en el turismo estructurado o mercantilizado no se observan. El vínculo en el destino permite un grado de seguridad, confianza y estabilidad que hace sentirse cómodo y tranquilo al turista.

A modo de reflexión final, se sostiene que la movilidad humana en el mundo contemporáneo es cada vez un patrón más frecuente de la vida social y que las formas de consumo turístico y cultural de los viajes se acoplan a esos cambios de paradigmas estableciendo constelaciones, redes y asociaciones que tejen los viajes en la modernidad.

\section{Bibliografía}

Backer, E., \& King, B. 2015. VFR Travel research : International Perspectives. Channel View Publications. Barretto, M. 2009. Interfaces entre turismo e migrações : uma abordagem epistemológica. Pasos Revista de Turismo y Patrimonio Cultural, 7, 1-11.

Bertoncello, R., \& Troncoso, C. 2014. La ciudad como objeto de deseo turístico: renovación urbana, cultura y turismo en Buenos Aires y Salta (Argentina). Gran Tour: Revista de Investigaciones Turisticas, 9, 4-26. 
Catalano, B. 2018. Prácticas socio-culturales de los turistas : un abordajecomparativo sobre los circuitos turísticos en la ciudad de Buenos Aires. Gran Tour: Revista de Investigaciones Turísticas, 18, 40-57.

Catalano, B. 2019. Movilidad turística e integración : teoría y métodos para su abordaje. Quid 16, Revista de Áreas de Estudios Urbanos Del Instituo de Investigaciones Gino Germani, 259-280.

Coles, T., Hall, C. M., \& Duval, D. T. 2006. Sobre el turismo y la movilidad en tiempos de movimiento y conjetura posdisciplinar. Política y Sociedad, 42(1), 85-99. http://revistas.ucm.es/index.php/POSO/ article/view/24110

Cordero Ulate, A. 2006. Nuevos ejes de acumulación y naturaleza. El caso del turismo. CLACSO, Consejo Latinoamericano de Ciencias Sociales.

Cresswell, T. 2008. Constellations of mobility. Institute of English Studies, 1-36.

Curcio, J., \& Luna, F. 2019. Impacto económico de los estudiantes internacionales en la Ciudad Autónoma de Buenos Aires (CABA).

De Santana Pinho, P. 2018. Mapping Diaspora: African American Roots Tourism in Brazil. UNC Press Book.

Dwyer, L., Seetaram, N., King, B., \& Forsyth, P. 2015. Is the Migration-Tourism Relationship only about VFR? Annals of Tourism Research, 46(3), 130-143. https://doi.org/10.1080/09669582.2014.9 86490.Using

Falero, A., \& Campodonico, R. 2014. El turismo bajo la lupa académica. UCUR.

Fernández Miranda, R. 2011. Viajar perdiendo el Sur. Crítica del turismo de masas en la globalización. Libros en acción.

Giddens, A. 1995. La constitución de la sociedad. Bases para la teoría de la estructuración. Amorrortu.

Glick Schiller, N., \& Salazar, N. B. 2013. Regimes of Mobility Across the Globe. Journal of Ethnic and Migration Studies, 39(2), 183-200.

Hall, C. M., \& Williams (Eds.). 2001. Tourism and Migration : New Relationships between Production and Consumption. Springer-science+busyness media.

Hannam, K., Sheller, M., \& Urry, J. 2006. Mobilities , Immobilities and Moorings. Mobilities, 1(1), 1-22. https://doi.org/10.1080/17450100500489189

Huete, R. 2009. Turistas que llegan para quedarse. Una explicación sociológica sobre la movilidad residencial. Universidad de Alicante.

Lanfant, M.-F. 1980. Introducción. El turismo en el proceso de internacionalización. Revista Internacional de Ciencias Sociales, 22(1), 14-45.

Larsen, J., Urry, J., \& Axhausen, K. 2006. Mobilities, Networks, Geographies. Ashgate Publishing Limited.

Larsen, J., Urry, J., \& Axhausen, K. W. 2007. Networks and tourism. Mobile Social Life. Annals of Tourism Research, 34(1), 244-262.

Lash, S., \& Urry, J. 1998. Economía de signos y espacios. Sobre el capitalismo de la posorganización. Amorrortu.

MacCannell, D. 2003. El turista. Una nueva teoría de la clase ociosa. Melusina.

Margulis, M. 2006. Ideología, fetichismo de la mercancía y reificación. Estudios Sociológicos El Colegio de México, XXIV, 31-64.

Mayer, L., \& Catalano, B. 2018. Internacionalización de la educación y movilidad : reflexiones a partir del caso argentino. Universitas. Revista de Ciencias Sociales y Humanas., 29, 19-41.

Merriman, P. 2014. Rethinking Mobile Methods. Mobilities, 9(2), 167-187.

Modolo, V. 2012. Política migratoria regional . El caso de la residencia Mercosur (2002-2011). Revista Aportes Para La Integración Latinoamericana, 40-58.

Murmis, M., \& Feldman, S. 2002. Formas de sociabilidad y lazos sociales. In Sociedad y sociabilidad en la Argentina de los 90 (p. 233). Biblos.

Novick, S. 2010. Migraciones y Mercosur: una relación inconclusa. In PhD Proposal (Vol. 1). Catálogos.

Otero, A. 2009. Las movilidades del turismo y las migraciones de amenidad: problemáticas y contradicciones en el desarrollo de centros turísticos de montaña. Revista de Geografía Norte Grande, 92, 75-92.

Schutz, A. 2003. Estudios sobre teoría social (1 A (Ed.)). Amorrortu.

Schutz, A. 2008. El problema de la realidad social (2da ed.). Amorrortu.

Shani, A., \& Uriely, N. 2012. VFR tourism. The Host Experience. Annals of Tourism Research, 39(1), 421-440. https://doi.org/10.1016/j.annals.2011.07.003

Sheller, M., \& Urry, J. 2004. Tourism Mobilities. Places to play, places in play (M. Sheller \& J. Urry (Eds.)). Routledge.

Smith, V. 1989. Anfitriones e invitados. Antropología del turismo. Endymion.

Strauss, A., \& Corbin, J. 2002. Bases de la investigación cualitativa: técnicas y procedimientos para desarrollar la teoría fundamentada. 
Tribe, J. 2011. The economics of recreation, leisure and tourism (4th ed.). Elsevier.

Urry, J. 2000. Sociologies: Sociology beyond Societies (J. Urry (Ed.)). Routledge.

Urry, J. 2002. Mobility and Proximity. 36(2), 255-274.

Vellas, F. 2004. Economía y política del turismo internacional. Síntesis.

Watson, G. L., \& Kopachevsky, J. P. 1994. Interpretations of tourism as commodity. Annals of Tourism Research, 21(3), 643-660. https://doi.org/10.1016/0160-7383(94)90125-2

White, N. R., \& White, P. B. 2007. Home and away. Tourists in a Connected World. Annals of Tourism Research, 34(1), 88-104. https://doi.org/10.1016/j.annals.2006.07.001

Williams, A. M., \& Hall, C. M. 2000. Tourism and migration: New relationships between production and consumption. Tourism Geographies, 2(1), 5-27. https://doi.org/10.1080/146166800363420

\section{Notas}

1 Se concibe como unidad de análisis la noción de práctica turística dado que preexiste una identidad de condiciones que reproducen sistemas de disposiciones semejantes a través de obras que se repiten en usos y costumbres y el significado y representaciones que los turistas tienen sobre sus mismas.

2 Los informantes son los nexos, es decir los familiares y/o amigos que los turistas vienen a visitar, mientras que los turistas, se corresponden a las diversas modalidades, es decir, los tradicionales y los VFA, con el fin de poder efectuar la diferenciación entre las dimensiones analizadas.

3 Según Hall and Williams (2001) "This is necessarily an oversimplification of course because there are other reason for visits to family and friends"

4 Encuesta de Turismo Internacional

5 Por "motivo principal del viaje" se entiende al motivo que determina la realización del viaje. Se habla de motivo principal porque es frecuente encontrar situaciones en las que se combinan más de un motivo: ocio y negocios, visita a familiares y estudio, etc.

6 Visita a familiares o amigos: se incluyen también aquí las visitas efectuadas para cuidar enfermos o asistir a exequias de familiares o amigos. (INDEC/MINTUR).

7 A partir del año 2011 en se efectúa una modificación en la ETI a partir de la cual se incorpora la categoría de "estudio" en motivaciones y se desagrega la categoría "Negocios" diferenciando los viajes para asistir conferencias y congresos de otros negocios. A su vez se le agrega la "recreación" al primer motivo.

8 Es necesario aclarar que los análisis sobre la llegada de turistas no residentes se realiza en base a los registros obtenidos en las vías aéreas y fluviales, es decir, no se tiene en cuenta la vía terrestre, ya que no se dispone de esos datos desagregados.

$9 \quad$ En la obra "Turistas que llegan para quedarse. Una explicación sociológica sobre la movilidad residencial" (2009) la autora Raquel Huete aborda la forma de movilidad que se denomina residencial, sus motivaciones, sus efectos y las relaciones existentes entre los turistas y los habitantes del lugar, los cambios sociales que provoca este fenómeno y las complejidades que atañen a esta forma híbrida entre turismo y migraciones.

10 Igualmente no siempre sucede que la conexión es con el migrante (Dwyer et al., 2015), puede darse que el turista visite a un amigo residente nativo o no, que haya conocido en otro sitio o en su lugar de origen, o en un evento, etc.

11 Según esta visión el turismo como mercancía revela que la reificación es un modo en el que la gente tiene necesidad de producir: las relaciones sociales reales entre la gente imita la apariencia socialmente necesaria de las relaciones entre las cosas. Asimismo se manifiesta otro tipo de alienación que toma forma de opciones restringidas enmarcadas por una "puesta en escena" un patrón de consumo - un fetichismo si se quiere - en la que el tema singular de la posesión y exhibición tiene prioridad sobre todos los demás la conducta social (Watson \& Kopachevsky, 1994, p. 649).

12 Organización Mundial del Turismo

13 Ministerio de Turismo de la Nación - República Argentina, es el principal organismo de aplicación en materia de turismo a nivel nacional.

14 Según la ETI, el motivo que determina la realización del viaje comprende las siguientes opciones de elección: vacaciones y ocio, visita a familiares o amigos, negocios, congresos y otros, que incluyen acontecimientos religiosos, personas que vienen con el fin de comprar todo tipo de mercaderías para uso personal o para regalo (excluye compras para reventa u objetos suntuosos).

15 Para ello se ha comparado la dimensión socialización y las actividades que realizan los turistas VFA con otros tipos de turistas.

16 Cabe la aclaración de que Almagro es un barrio que no se encuentra dentro del casco turístico de la ciudad, no es frecuentado en su mayoría por turistas sino más bien por ciudadanos. Además posee una oferta de bares culturales y restaurantes.

17 Se denomina nexo de turistas a un tipo de informante clave, generalmente es el familiar y/o amigo que el turista visita en el destino

18 En "Mobility and proximity", Urry (2002) hace referencia a que a pesar de que los avances en las tecnologías nos facilitan las comunicaciones y el estar conectado con personas distantes, la gente persiste utilizando la movilidad física a pesar de las facilidades que brinda internet. Según el autor, en ciertos casos la interacción presencial es irremplazable, dado que aspectos tales como el contacto visual, expresiones corporales, gestos y lenguaje corporal no se manifiestan de la misma manera que en las comunicaciones virtuales.

19 Para usar la categorías de motivos que se establece en la encuesta ETI.

Recibido:

Reenviado: 